\title{
Interaction Potential between Parabolic Rotator and an Outside Particle
}

\author{
Dan Wang, Yajun Yin, Jiye Wu, Xugui Wang, and Zheng Zhong \\ Department of Engineering Mechanics, School of Aerospace, Tsinghua University, Beijing 100084, China
}

Correspondence should be addressed to Yajun Yin; yinyj@mail.tsinghua.edu.cn

Received 29 April 2014; Accepted 19 June 2014; Published 7 July 2014

Academic Editor: Shaoyun Fu

Copyright (C) 2014 Dan Wang et al. This is an open access article distributed under the Creative Commons Attribution License, which permits unrestricted use, distribution, and reproduction in any medium, provided the original work is properly cited.

At micro/nanoscale, the interaction potential between parabolic rotator and a particle located outside the rotator is studied on the basis of the negative exponential pair potential $(1 / R)^{n}$ between particles. Similar to two-dimensional curved surfaces, we confirm that the potential of the three-dimensional parabolic rotator and outside particle can also be expressed as a unified form of curvatures; that is, it can be written as the function of curvatures. Furthermore, we verify that the driving forces acting on the particle may be induced by the highly curved micro/nano-parabolic rotator. Curvatures and the gradient of curvatures are the essential elements forming the driving forces. Through the idealized numerical experiments, the accuracy of the curvature-based potential is preliminarily proved.

\section{Introduction}

In recent years, various phenomena at micro/nanoscales increasingly draw people's attention. The highly curved spaces widely exist at micro/nanoscales. As a typical form, curved surfaces are objects studied in differential geometry. In addition, there is another space form, that is, the curved surface body. A curved surface body is a three-dimensional body. In this sense, it is outside the range of curved spaces. Nevertheless, when the size scale we study is small enough, the large specific surface area and the highly curved outer surface will powerfully connect curved surface body with curved space.

Similar to curved surfaces, curved surface bodies widely exist at micro/nanoscales. In biological system, organic particles may be transported between cells by the tubular conduits of tunneling nanotubes [1] (Figure 1). Human fibroblasts extend by sensing the fiber curvatures [2]. Inward curvatures of the plasma membrane may cause BAR-domain proteins to release Rac [2,3]. Silicone oil droplets on conical surfaces will move spontaneously towards the end with bigger diameter [4]. Through the interaction between the tip (Figure 2) and the tested object [5], atomic force microscope can probe the morphologies of surfaces [6]. All these cases involve the interaction of the curved surface bodies.
Previously, based on the pair-potential of particles $\left((1 / R)^{n}\right)$, Yin et al. [7] studied the interaction between micro/nano-curved surface and a particle located outside the surface. Wu et al. [8,9] explored the interaction between micro/nano-curved surface and a particle located inside the surface. Their researches verify that interaction between highly curved surface and particle can be expressed as the function of curvatures. Then they give the following propositions. (a) At micro/nanoscale, the highly curved surface can induce driving forces. (b) The essential elements that form the driving forces are curvatures and the gradient of curvatures.

Previous studies inspire us to raise questions. Will the interaction potential between curved surface body and particle be expressed as the function of curvatures? Whether the curved surface of the curved surface body can induce the driving forces as well? This paper will give the answers.

\section{Interaction between Semi-Infinite Plane Body and a Particle Located outside the Plane}

As a prelude, we review the interaction between semi-infinite plane body and a particle located outside the plane, which has already existed in the flat space. 
(a)

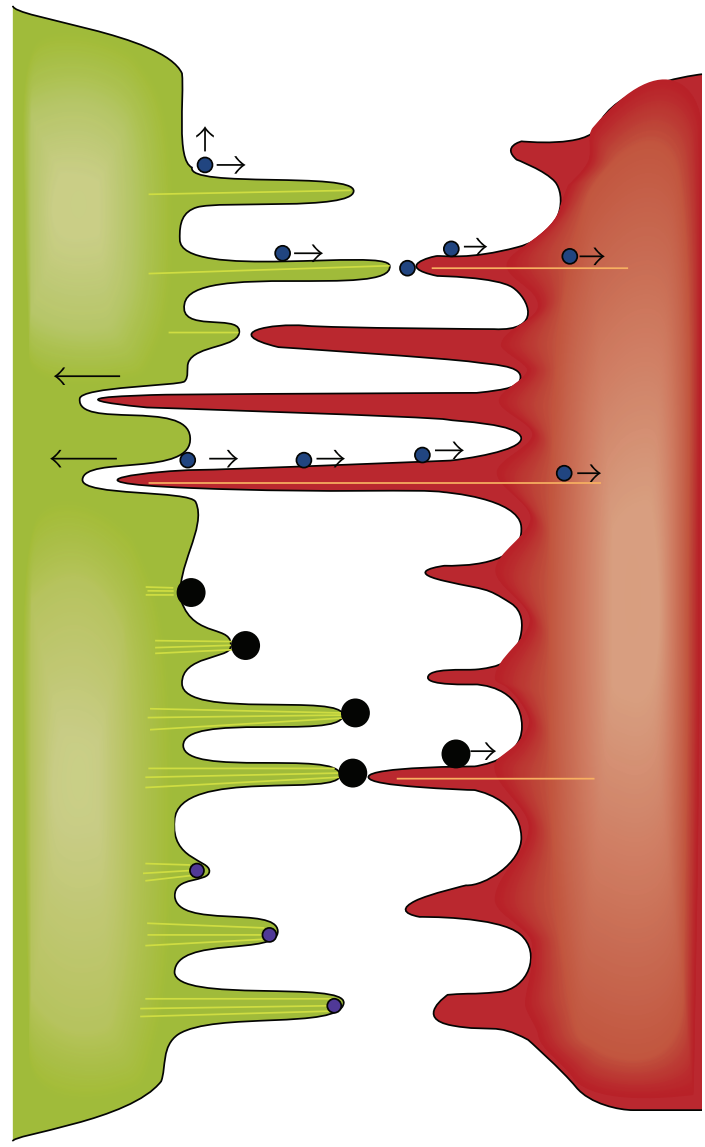

FIGURE 1: The transportation of organic particles between cells by tunneling nanotubes [1].
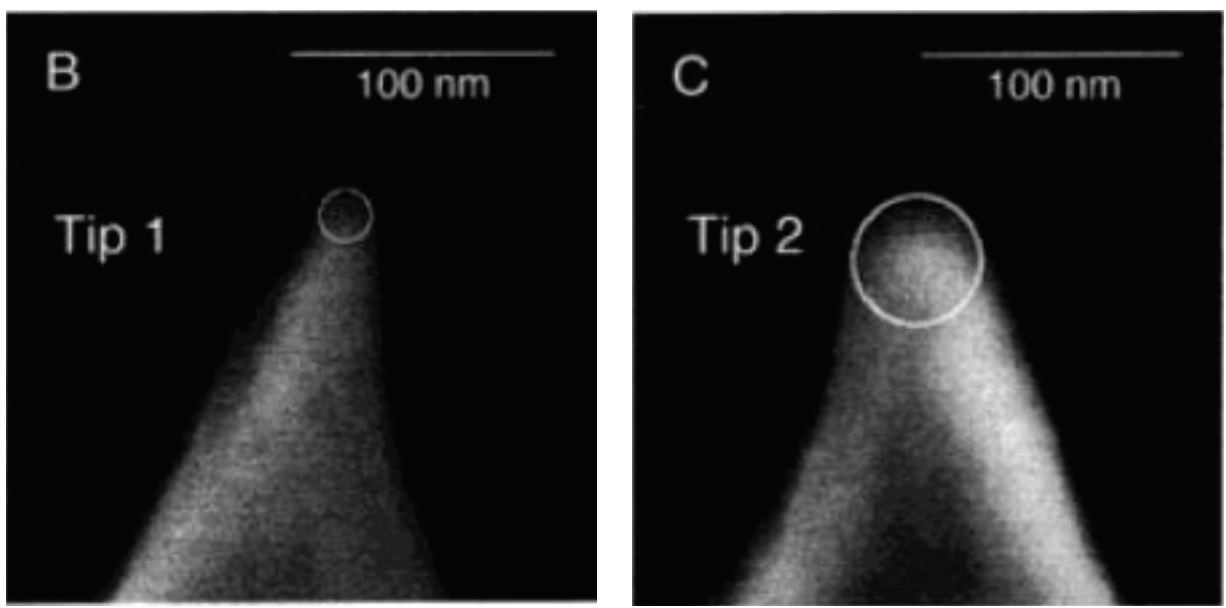

FIGURE 2: Atomic force microscopes with sharp probe (B) and blunt probe (C) [5].

Semi-infinite plane body belongs to the threedimensional Euclid space. Thus, the interaction between it and an outside particle is a classical mechanical problem. The potential can be found in the book written by Israelachvili [10]. As shown in Figure 3, if we assume that the pairpotential between particles has the form of $u(r)=C / r^{n}$, the number density of molecules in semi-infinite plane body is $\rho$, and the nearest distance between the particle and the plane is $h$, then the interaction potential of the particle $p$ and semi-infinite body can be written as

$$
\bar{U}_{n}=\frac{2 \pi \rho C}{(n-2)(n-3) h^{n-3}}, \quad n \geq 4 .
$$




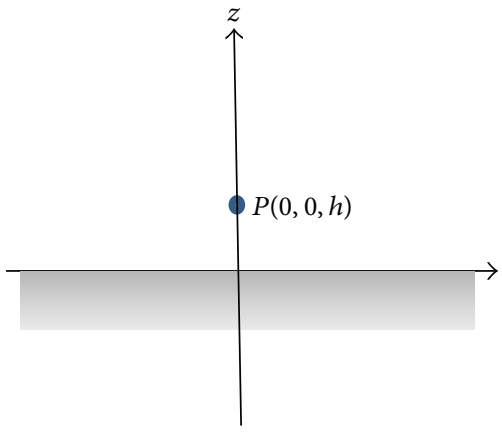

Figure 3: The interaction between semi-infinite plane body and an outside particle.

If the surface of the plane body shown in Figure 3 bends, it will become a curved surface body. Hence, semi-infinite plane body is not only a particular case of the curved surface body, but also the foundation for further studies.

\section{Classification of Parabolic Rotators}

Previously, researchers have studied the interaction between certain curved surface body and a particle, like a sphere and a cylinder [11, 12]. As a preliminary test, this paper will study another simple case, that is, the interaction between a parabolic rotator and a particle located outside the rotator.

According to the bending direction, parabolic rotators can be divided into two categories, that is, convex parabolic rotator and concave parabolic rotator (Figure 4). Obviously, these two kinds of parabolic rotators are different when they interact with an outside particle. From this perspective, these two cases should be studied separately.

Whatever it is convex or concave, the biggest commonality of two kinds of parabolic rotators is that they have similar curved surfaces, that is, the revolutionary paraboloid. In this sense, it is possible to study two kinds of parabolic rotators uniformly. A unified coordinate system is used to ensure the uniformity.

As shown in Figures 5 and 6, a local Cartesian coordinate system $O-x y z$ is built at the vertex $O$ of the parabolic rotator. The $x-y$ plane is the tangent plane of the parabolic rotator at the vertex $O$. The function of the revolutionary paraboloid for the convex and concave rotators can be uniformly expressed as $z=(c / 2)\left(x^{2}+y^{2}\right)$ in such coordinate system. By the differential geometry [13], the principal curvatures at the vertex $O$ of the revolutionary paraboloid are $c_{1}=c_{2}=c$ with $c>0$ in the illustrated coordinate system.

Assuming that the outside particle $p$ is located on the axis $z$ and has a distance $h$ to the vertex $O$, the coordinate of the particle $p$ is $(0,0,-h)$ for convex parabolic rotator and $(0,0, h)$ for concave parabolic rotator. To ensure uniformity, we write the coordinate of the particle $p$ as $\left(0,0, z_{p}\right)$. Therefore, $z_{p}=-h$ corresponds to convex parabolic rotator and $z_{p}=h$ corresponds to concave parabolic rotator. Define the dimensionless coordinates as

$$
\widetilde{z}_{p}=\frac{z_{p}}{h}
$$

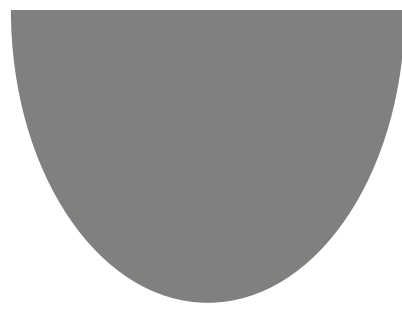

(a)

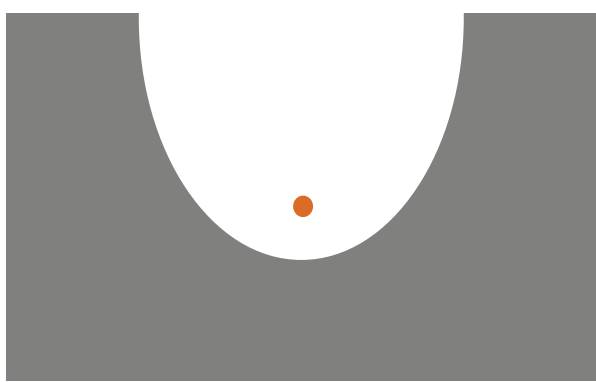

(b)

FIGURE 4: (a) Convex parabolic rotator and an outer particle; (b) concave parabolic rotator and an outer particle.

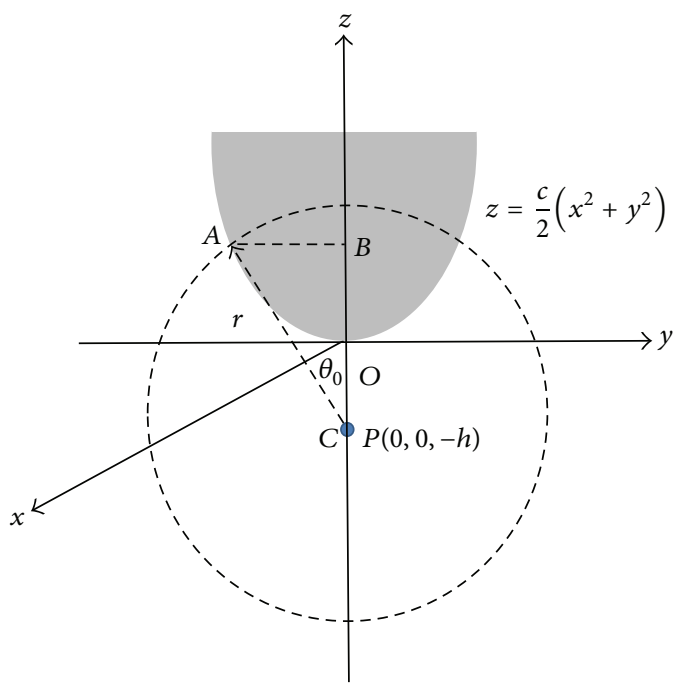

FIGURE 5: The coordinate system of convex parabolic rotator and an outside particle.

Obviously, there are only two values for $\widetilde{z}_{p} \cdot \widetilde{z}_{p}=-1$ corresponds to convex parabolic rotator, and $\widetilde{z}_{p}=+1$ corresponds to concave parabolic rotator.

\section{Interaction Potential between Parabolic Rotator and a Particle}

4.1. The General Formulation of the Potential. In order to derive the interaction potential between the particle $p$ and the parabolic rotator, we draw a sphere with the center at particle 


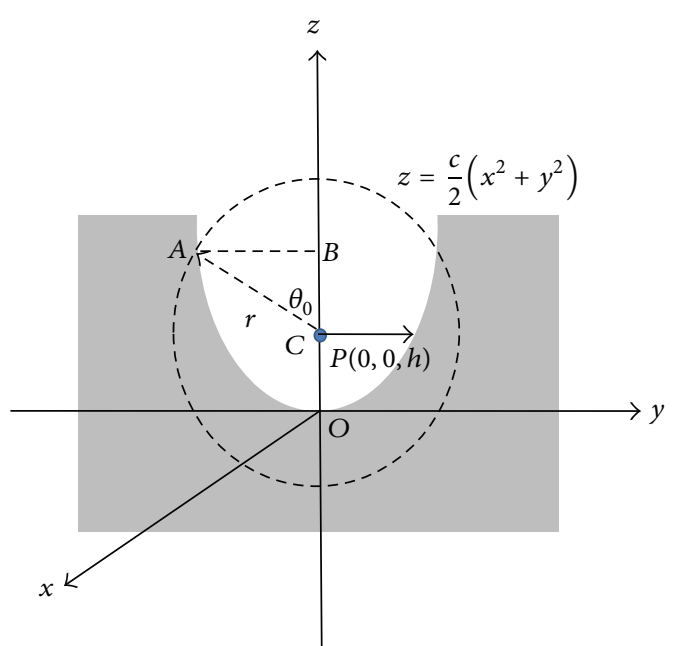

FIGURE 6: The coordinate system of concave parabolic rotator and an outside particle.

$p$ and the radius $r$. The intersection part of spherical surface and parabolic rotator is an arc surface with the area $S$. Then the potential between the particle and parabolic rotator is

$$
U_{n}=\int_{h}^{\infty} \frac{C \rho_{v} S}{r^{n}} d r
$$

Equation (3) is valid for both convex parabolic rotator and concave parabolic rotator.

4.2. The Uniform Formulation of the Area of the Arc Surface. The area $S$ in (3) can be determined as follows. For convex parabolic rotator (Figure 5), we have

$$
\widehat{S}=\int_{0}^{2 \pi} d \varphi \int_{0}^{\theta_{0}} r^{2} \sin \theta d \theta=2 \pi r^{2}\left(1-\cos \theta_{0}\right) .
$$

The spherical center angle $\theta_{0}$ satisfies

$$
\cos \theta_{0}=\frac{z+h}{r}
$$

In triangle $\mathrm{ABC}$, we have

$$
x^{2}+y^{2}+(z+h)^{2}=r^{2} .
$$

By combining $z=(c / 2)\left(x^{2}+y^{2}\right)$ with (5) and (6), we get

$$
\cos \theta_{0}=\frac{\sqrt{(2 h / c)+\left(1 / c^{2}\right)+r^{2}}-(1 / c)}{r} .
$$

Substitution of (7) into (4) leads to

$$
\widehat{S}=2 \pi r\left[r+\frac{1}{c}-\frac{1}{c} \sqrt{1+2 h c+(c r)^{2}}\right] .
$$

For concave parabolic rotator (Figure 6), there is

$$
\begin{gathered}
\breve{S}=4 \pi r^{2}-\int_{0}^{2 \pi} d \varphi \int_{0}^{\theta_{0}} r^{2} \sin \theta d \theta=2 \pi r^{2}\left(1+\cos \theta_{0}\right), \\
\cos \theta_{0}=\frac{z-h}{r} .
\end{gathered}
$$

In triangle $\mathrm{ABC}$, there is

$$
x^{2}+y^{2}+(z-h)^{2}=r^{2} .
$$

By combing $z=(c / 2)\left(x^{2}+y^{2}\right)$ with (10) and (11), we get

$$
\cos \theta_{0}=\frac{\sqrt{-(2 h / c)+\left(1 / c^{2}\right)+r^{2}}-(1 / c)}{r} .
$$

Substitution of (12) into (9) gives

$$
\breve{S}=2 \pi r\left[r-\frac{1}{c}+\frac{1}{c} \sqrt{1-2 h c+(c r)^{2}}\right] .
$$

Obviously, with the dimensionless coordinate $\widetilde{z}_{p},(8)$ and (13) can be uniformly written as

$$
S=2 \pi r\left[r-\frac{1}{\widetilde{z}_{p} c}+\frac{1}{\widetilde{z}_{p} c} \sqrt{1-2 h \tilde{z}_{p} c+\left(\widetilde{z}_{p} c r\right)^{2}}\right] .
$$

4.3. Curvature-Based Interaction Potential between Particle and Parabolic Rotator. By substituting (14) into (3), we obtain the interaction potential between the particle $p$ and convex and concave parabolic rotators uniformly:

$$
\begin{aligned}
& U_{n}=2 \pi \rho_{v} C \\
& \quad \times \int_{h}^{\infty} \frac{1}{r^{n-1}} \\
& \quad \times\left[r-\frac{1}{\widetilde{z}_{p} c}+\frac{1}{\widetilde{z}_{p} c} \sqrt{1-2 h \widetilde{z}_{p} c+\left(\widetilde{z}_{p} c r\right)^{2}}\right] d r .
\end{aligned}
$$

The objective of this section is to express (15) as an explicit function of curvature. The technique is mature, that is, the series expansion for small parametric variable [14]. The distance $h$ is used as the characteristic length in dimensionless transformations:

$$
\widetilde{c}=c h, \quad \widetilde{r}=\frac{r}{h} .
$$

Then (15) can be written as

$$
\begin{aligned}
U_{n}= & \frac{2 \pi \rho_{v} C}{h^{n-3}} \\
& \times \int_{1}^{\infty} \frac{1}{\widetilde{r}^{n-1}} \\
& \times\left[\tilde{r}-\frac{1}{\widetilde{z}_{p} \widetilde{c}}+\frac{1}{\widetilde{z}_{p} \widetilde{c}} \sqrt{1-2 \widetilde{z}_{p} \widetilde{c}+\left(\widetilde{z}_{p} \widetilde{c} \widetilde{r}\right)^{2}}\right] d \widetilde{r}
\end{aligned}
$$

In (17), let

$$
f(\widehat{c})=\sqrt{1-2 \widehat{c}+(\widehat{c} \widetilde{r})^{2}} .
$$

Here $\widehat{c}=\widetilde{z}_{p} \widetilde{c}$ and $f(\widehat{c})$ is the continuously differentiable function of $\widehat{c}$. Suppose that the distance $h$ is a small quantity 
TABLE 1: The error of curvature-based potential between the convex parabolic rotator and the particle $(n=6)$.

\begin{tabular}{|c|c|c|c|c|}
\hline$\widetilde{c}$ & $\left.\widetilde{\widetilde{U}}_{6}\right)_{\text {numerical }}$ & $\left.\widetilde{\widetilde{U}}_{6}\right)_{\text {polynomial }}$ & $\delta=$ & $\mid \frac{\left(\widetilde{\widehat{U}}_{6}\right)_{\text {polynomial }}-\left(\widetilde{\widehat{U}}_{6}\right)_{\text {numerical }}}{\left.\widetilde{\widetilde{U}}_{6}\right)_{\text {numerical }}}$ \\
\hline 0.5 & 0.554354020074120 & 0.625000000000000 & & 0.127438382996544 \\
\hline 0.4 & 0.609849704658992 & 0.640000000000000 & & 0.049438894715652 \\
\hline 0.3 & 0.677316303948916 & 0.685000000000000 & & 0.011344324662327 \\
\hline 0.1 & 0.866023611886837 & 0.865000000000000 & & 0.001181967642437 \\
\hline 0.05 & 0.929014971306476 & 0.928750000000000 & & $.852174772848038 e-004$ \\
\hline 0.03 & 0.956428713838380 & 0.956350000000000 & & $.229974407998883 e-005$ \\
\hline 0.01 & 0.985151878051310 & 0.985150000000000 & & $.906357133214219 e-006$ \\
\hline 0.005 & 0.992534562174735 & 0.992537500000000 & & $.959922381342625 e-006$ \\
\hline 0.001 & 0.998496854809661 & 0.998501500000000 & & $.652183245473563 e-006$ \\
\hline 0.0001 & 0.999845094980028 & 0.999850015000000 & & $.920782226301011 e-006$ \\
\hline
\end{tabular}

compared to the curvature radius at the vertex of parabolic rotator. Therefore, the dimensionless curvatures satisfy $\widetilde{c} \ll 1$ and $|\widehat{c}| \ll 1$. Take $\widehat{c}$ as the small parametric variable and use the method of series expansion [14]. Function $f(\widehat{c})$ can be expanded as Taylor's series of small parametric variable $\widehat{c}$ :

$$
\begin{aligned}
f(\widehat{c}) \approx & f(0)+\left(\left.\frac{\partial f}{\partial \widehat{c}}\right|_{\widehat{c}=0}\right) \widehat{c}+\frac{1}{2 !}\left(\left.\frac{\partial^{2} f}{\partial \widehat{c}^{2}}\right|_{\widehat{c}=0}\right) \widehat{c}^{2} \\
& +\frac{1}{3 !}\left(\left.\frac{\partial^{3} f}{\partial \widehat{c}^{3}}\right|_{\widehat{c}=0}\right) \widehat{c}^{3}+\frac{1}{4 !}\left(\left.\frac{\partial^{4} f}{\partial \widehat{c}^{4}}\right|_{\widehat{c}=0}\right) \widehat{c}^{4}+\cdots \\
& +\frac{1}{m !}\left(\left.\frac{\partial^{m} f}{\partial \widehat{c}^{m}}\right|_{\widehat{c}=0}\right) \widehat{c}^{m}+O\left(\widehat{c}^{m}\right) .
\end{aligned}
$$

We omit the terms whose orders are higher than 3 to get the polynomial of variable $\widetilde{c}$ :

$$
\begin{aligned}
f(\widehat{c}) & \approx f(0)+f^{\prime}(0) \widehat{c}+\frac{f^{\prime \prime}(0)}{2 !} \widehat{c}^{2}+\frac{f^{\prime \prime \prime}(0)}{3 !} \widehat{c}^{3}+O\left(\widehat{c}^{3}\right) \\
& =1-\widehat{c}+\frac{1}{2}\left(\widetilde{r}^{2}-1\right) \hat{c}^{2}+\frac{1}{2}\left(\widetilde{r}^{2}-1\right) \widehat{c}^{3} .
\end{aligned}
$$

Substitution of (20) into (17) leads to the uniform polynomial of curvature-based potential:

$$
U_{n}=\bar{U}_{n}\left[1+\frac{n-3}{n-4} \widetilde{z}_{p} \widetilde{c}+\frac{n-3}{n-4}\left(\widetilde{z}_{p} \widetilde{c}\right)^{2}\right] .
$$

Equation (21) requires $n \geq 5$. The value of index $n$ is determined by the expanding order of $\widehat{c}$ in (19). If the series is expanded to $\widehat{c}^{3}$, then $n \geq 4$ is needed.

Although parabolic rotator is three-dimensional body, (21) indicates that the interaction potential between the rotator and the particle is still decided by the curvature $c$. If $c \rightarrow 0$, we have $U_{n} \rightarrow \bar{U}_{n}$; that is, the interaction potential $U_{n}$ will degenerate to $\bar{U}_{n}$. Once the flat surface is curved, the interaction potential is no longer $\bar{U}_{n}$, and a modified term of curvatures must be involved. The more curved the space is, the more significant the influence of modified term of curvatures is.

\section{Numerical Verification}

What is the accuracy of the curvature-based potential of polynomial? The answer will be provided through numerical verification.

First, both sides of curvature-based potential of polynomial (21) are divided by $\bar{U}_{n}$ to get the dimensionless form:

$$
\left(\widetilde{U}_{n}\right)_{\text {polynomial }}=\frac{U_{n}}{\bar{U}_{n}}=1+\frac{n-3}{n-4} \widetilde{z}_{p} \widetilde{c}+\frac{n-3}{n-4}\left(\widetilde{z}_{p} \widetilde{c}\right)^{2}
$$

Then both sides of (17) are divided by $\bar{U}_{n}$ to get the dimensionless potential of integral form:

$$
\begin{aligned}
& \left(\widetilde{U}_{n}\right)_{\text {numerical }} \\
& =(n-2)(n-3) \\
& \quad \times \int_{1}^{\infty} \frac{1}{\widetilde{r}^{n-1}} \\
& \quad \times\left[\tilde{r}-\frac{1}{\widetilde{z}_{p} \widetilde{c}}+\frac{1}{\widetilde{z}_{p} \widetilde{c}} \sqrt{1-2 \widetilde{z}_{p} \widetilde{c}+\left(\widetilde{z}_{p} \widetilde{c} \widetilde{r}\right)^{2}}\right] d \widetilde{r} .
\end{aligned}
$$

Finally, $\left(\widetilde{U}_{n}\right)_{\text {numerical }}$ in $(23)$ and $\left(\widetilde{U}_{n}\right)_{\text {polynomial }}$ in $(22)$ are compared, and the accuracy of (22) may be estimated:

$$
\delta=\left|\frac{\left(\widetilde{U}_{n}\right)_{\text {polynomial }}-\left(\widetilde{U}_{n}\right)_{\text {numerical }}}{\left(\widetilde{U}_{n}\right)_{\text {numerical }}}\right| \times 100 \% .
$$

In the numerical calculations, we will take $n=6$. The errors of curvature-based potential of convex and concave parabolic rotators are listed in Tables 1 and 2, respectively. From these we can see that the curvature-based potential has enough accuracy when $\tilde{c}$ is a small quantity. 
TABLE 2: The error of curvature-based potential between the concave parabolic rotator and the particle $(n=6)$.

\begin{tabular}{lcc|c}
\hline$\tilde{c}$ & $\left(\widetilde{U}_{6}\right)_{\text {numerical }}$ & $\left(\widetilde{U}_{6}\right)_{\text {polynomial }}$ & $\delta=\mid \frac{\left(\widetilde{U}_{6}\right)_{\text {polynomial }}-\left(\widetilde{U}_{6}\right)_{\text {numerical }} \mid}{\left(\widetilde{U}_{6}\right)_{\text {numerical }}}$ \\
\hline 0.5 & 1.999990099763677 & 2.125000000000000 & 0.062505259526582 \\
0.4 & 1.767008983006020 & 1.840000000000000 & 0.041307666059404 \\
0.3 & 1.548796643131850 & 1.585000000000000 & 0.023375151946961 \\
0.1 & 1.162688575661165 & 1.165000000000000 & 0.001987999527320 \\
0.05 & 1.078359520840800 & 1.078750000000000 & $3.621048005354316 e-004$ \\
0.03 & 1.046242770650530 & 1.046350000000000 & $1.024899310924430 e-004$ \\
0.01 & 1.015137871366782 & 1.015150000000000 & $1.194776942179518 e-005$ \\
0.005 & 1.007530503256329 & 1.007537500000000 & $6.944448479219859 e-006$ \\
0.001 & 1.001496244320401 & 1.001501500000000 & $5.247827567111332 e-006$ \\
0.0001 & 1.000145034778507 & 1.000150015000000 & $4.979499292623452 e-006$ \\
\hline
\end{tabular}

\section{Discussions}

6.1. Local Properties of the Curvature-Based Potential. The integrand in (3) is

$$
g(\tilde{r}, \widetilde{c})=\frac{1}{\widetilde{r}^{n-1}}\left[\widetilde{r}-\frac{1}{\widetilde{z}_{p} \widetilde{c}}+\frac{1}{\widetilde{z}_{p} \widetilde{c}} \sqrt{1-2 \widetilde{z}_{p} \widetilde{c}+\left(\widetilde{z}_{p} \widetilde{c} \widetilde{r}\right)^{2}}\right] .
$$

The domain of integration is $[h, \infty)$. Hence the interaction between parabolic rotator and the particle reflects the global properties of the system. However, in the viewpoint of physics, the short-range interaction pair-potential determines that the particle $p$ mainly interacts with the nearest zone of curved surface body. Thus, from the mathematical point of view, the distribution of integrand $g(\widetilde{r}, \widetilde{c})$ is of highly local properties, which can be further proved as follows. For convex and concave parabolic rotators, there are

$$
\begin{aligned}
& \widehat{g}(\widetilde{r}, \widetilde{c})=\frac{1}{\widetilde{r}^{n-1}}\left[\widetilde{r}+\frac{1}{\widetilde{c}}-\frac{1}{\widetilde{c}} \sqrt{1+2 \widetilde{c}+(\widetilde{c} \widetilde{r})^{2}}\right], \\
& \breve{g}(\widetilde{r}, \widetilde{c})=\frac{1}{\widetilde{r}^{n-1}}\left[\widetilde{r}-\frac{1}{\widetilde{c}}+\frac{1}{\widetilde{c}} \sqrt{1-2 \widetilde{c}+(\widetilde{c} \widetilde{r})^{2}}\right] .
\end{aligned}
$$

The distribution diagrams are shown in Figures 7 and 8. We can see that the values of the integrand mainly distribute in the region of the dimensionless radius $\tilde{r} \in[1,3]$. Beyond this region the integrand decreases to zero rapidly.

Moreover, we can interpret the mechanism in Figures 7 and 8 concretely. Along with the increase of the radius $\tilde{r}$, the area of arc surface cutoff by the sphere with center $p$ from the curved surface body gets larger. However, the pairpotential between particle $p$ and particles on the arc surface decreases sharply. Their product will reach the maximum when $\tilde{r}$ comes to a certain value. Later, the effect decreasing potential exceeds that of the increasing area, causing the integrand to decrease rapidly. This is the mathematical foundation for the local properties of the interaction between the particle and the parabolic rotator. Since the local particles of parabolic rotator are mostly distributed on the surface and the surface morphology is depicted by curvatures, the interaction between the particle and parabolic rotator is

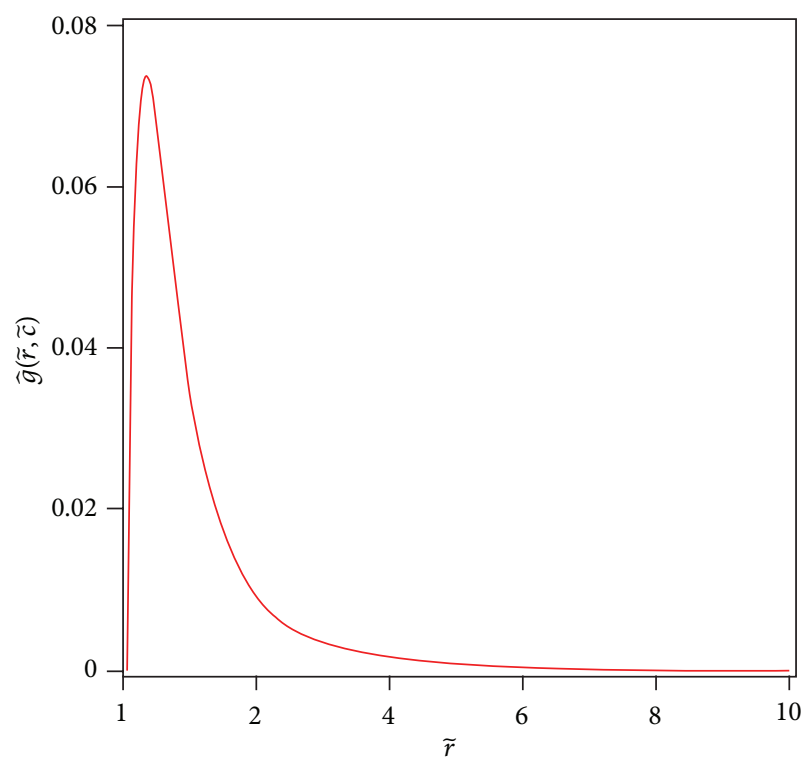

FIGURE 7: The distribution of $\widehat{g}(\widetilde{r}, \widetilde{c})$ along with $\widetilde{r}$ when $n=6, \widetilde{c}=$ 0.1 .

mainly determined by curvatures and can be written as the curvature-based form finally.

6.2. The Driving Force on the Particle. The dimensional form of the curvature-based potential in (21) is

$$
U_{n}=\bar{U}_{n}\left[1+\frac{n-3}{n-4} z_{p} c+\frac{n-3}{n-4}\left(z_{p} c\right)^{2}\right] .
$$

Supposing that the movement of the particle is restricted on the axis $z$, the driving force exerted on the particle in the $z$ direction isc

$$
\begin{aligned}
F_{z}= & -\frac{\partial U_{n}}{\partial z_{p}}=-\bar{U}_{n} \frac{n-3}{n-4} c\left[1+2 z_{p} c\right] \\
& -\frac{\partial \bar{U}_{n}}{\partial h} \frac{\partial h}{\partial z_{p}}\left[1+\frac{n-3}{n-4} z_{p} c+\frac{n-3}{n-4}\left(z_{p} c\right)^{2}\right] .
\end{aligned}
$$




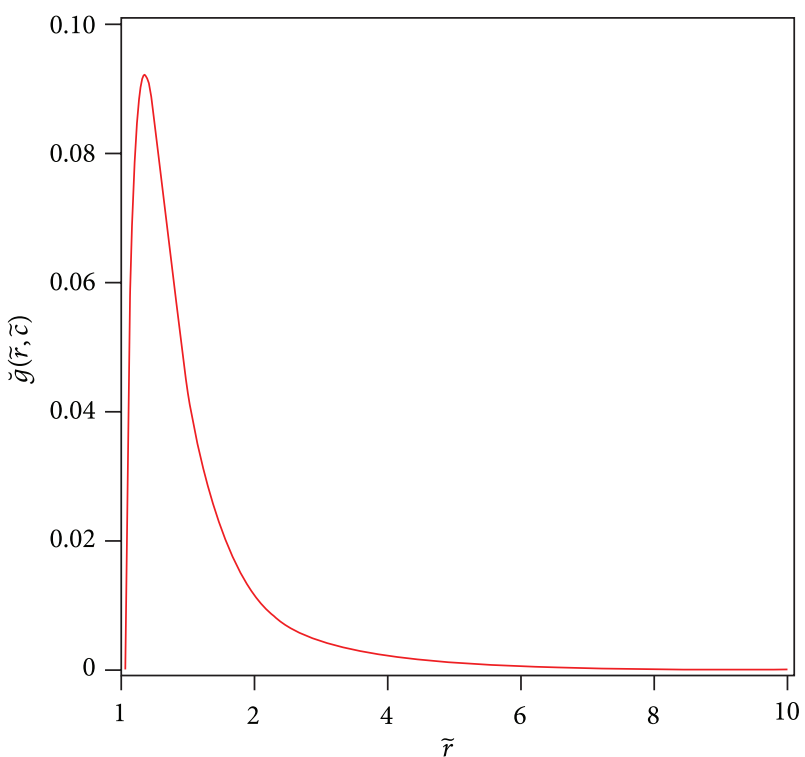

Figure 8: The distribution of $\breve{g}(\widetilde{r}, \widetilde{c})$ along with $\widetilde{r}$ when $n=6, \widetilde{c}=$ 0.1 .

Here there is $\partial h / \partial z_{p}= \pm 1$, and it takes minus sign for convex curved surface body and positive sign for concave curved surface body.

If $c \rightarrow 0$, that is, parabolic rotator generates on semifinite plane body, then (28) is

$$
F_{z}=-\frac{\partial \bar{U}_{n}}{\partial h} \frac{\partial h}{\partial z_{p}}
$$

Equation (29) is the driving force acting on the particle by semifinite plane body, which is a classical conclusion.

Once $c \rightarrow \infty$, then $F_{z} \rightarrow \infty$; that is, there are infinite forces acting on the particle if curvatures of vertex of parabolic body tend to infinity. Such vertex with infinite curvatures is a sharp singularity geometrically. Thus, the sharp singularity can induce strong driving force, which provides insight to understand a large amount of abnormal movements around singularities.

Specifically, if such driving force is attractive, strong effect of hydrophilic will cause water droplets come into being around singularities, which can help living organism to absorb water from environment (Figure 9). Otherwise, repulsive force will cause hydrophobic effect and help living organism to discharge water into environment.

The above analysis enhances previous propositions: curved spaces can induce driving forces. Definitely, curvatures can induce driving forces. By changing curvatures, driving forces acting on the particle can be changed, which means that it is possible to regulate movements of particles by noncontacting and geometrical methods.

If the particle $P$ is confined to the parallel curved surface with $h=$ Const. (Figure 10) and can only move along the generating line of the parallel curved surface, then the particle will sense the tangential force induced by the changing of curvatures. Parabolic body is a particular case of curved surface body, as principle curvatures at vertex are equal; that

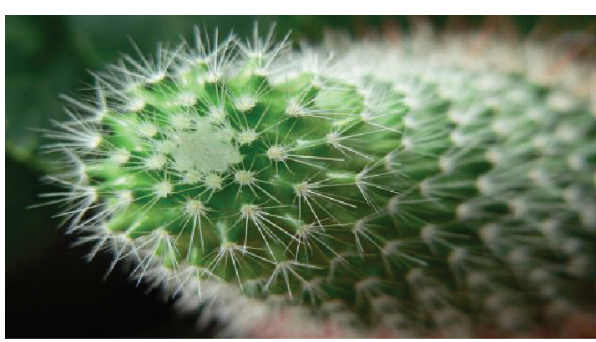

(a)

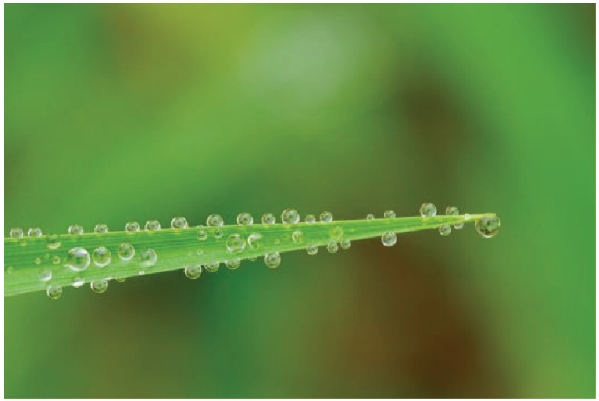

(b)

FIGURE 9: The driving force around singularities: hydrophilic or hydrophobic effect.

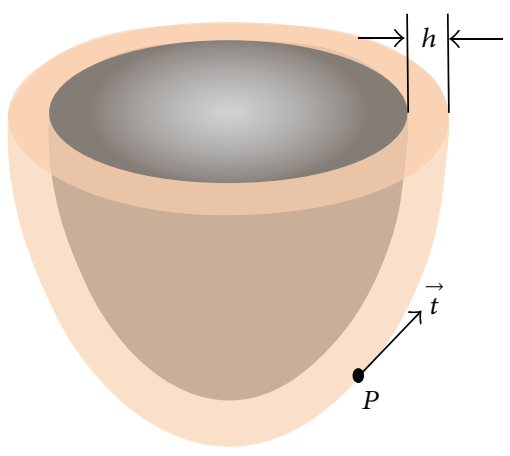

FIGURE 10: The movement of the particle induced by curvatures on the parallel surface.

is, $c_{1}=c_{2}=c$. But once the particle moves and deviates from the top of vertex, principle curvatures of two directions at the nearest point from the body to the particle come to unequal magnitude. Thus, in order to write the tangential force as a function of the gradient of curvatures, the interaction potential between the particle and general curved surface body should be studied, which will be shown in a subsequent paper.

\section{Conclusions}

Although the parabolic rotator at micro/nanoscale may be a particular case of curved surface bodies, the propositions deduced from it do not lose universality; that is, they are valid in curved surface bodies with any bending surfaces. In the succeeding papers, we will expand the propositions in this paper to general curved surface bodies. Based on 
the progress in this paper, we can say that the propositions derived in the curved surface and curved line will stay valid in the curved surface body as well. In other words, the proposition that micro/nano-curved spaces induce driving force is of universality. It also means that curvature is a crucial factor at micro/nanoscale and should be one of the cores in micro/nanomechanics.

\section{Conflict of Interests}

The authors declare that there is no conflict of interests regarding the publication of this paper.

\section{Acknowledgments}

This work was supported by the National Natural Sciences Foundation of China (Grant nos. 11072125, 11272175, 11102138, and 10602040) as well as the Natural Science Foundation of Jiangsu province (Grant nos. BK2011075 and BK20130910) and Foundation of Doctoral Supervisor.

\section{References}

[1] N. M. Sherer and W. Mothes, "Cytonemes and tunneling nanotubules in cell-cell communication and viral pathogenesis," Trends in Cell Biology, vol. 18, no. 9, pp. 414-420, 2008.

[2] V. Vogel and M. Sheetz, "Local force and geometry sensing regulate cell functions," Nature Reviews Molecular Cell Biology, vol. 7, no. 4, pp. 265-275, 2006.

[3] B. Habermann, "The BAR-domain family of proteins: a case of bending and binding?” EMBO Reports, vol. 5, no. 3, pp. 250-255, 2004.

[4] É. Lorenceau and D. Quéré, "Drops on a conical wire," Journal of Fluid Mechanics, vol. 510, pp. 29-45, 2004.

[5] D. J. Wold and C. D. Frisbie, "Fabrication and characterization of metal-molecule-metal junctions by conducting probe atomic force microscopy," Journal of the American Chemical Society, vol. 123, no. 23, pp. 5549-5556, 2001.

[6] C. Argento and R. H. French, "Parametric tip model and forcedistance relation for Hamaker constant determination from atomic force microscopy," Journal of Applied Physics, vol. 80, no. 11, pp. 6081-6090, 1996.

[7] Y. Yin, C. Chen, C. Lü, and Q. Zheng, "Shape gradient and classical gradient of curvatures: driving forces on micro/nano curved surfaces," Applied Mathematics and Mechanics, vol. 32, no. 5, pp. 533-550, 2011.

[8] J. Y. Wu, Y. J. Yin, X. G. Wang, and Q. S. Fan, "Interaction potential between micro/nano curved surface and a particle located inside the surface (I): driving forces induced by curvatures," Science China Physics, Mechanics and Astronomy, vol. 55, no. 6, pp. 1066-1076, 2012.

[9] J. Y. Wu, Y. J. Yin, K. Huang, and Q. S. Fan, "Interaction potential between micro/nano curved surface and a particle located inside the surface (II): numerical experiment and equipotential surfaces," Science China: Physics, Mechanics and Astronomy, vol. 55, no. 6, pp. 1077-1082, 2012.

[10] J. Israelachvili, Intermolecular and Surface Forces, Academic Press, London, UK, 2nd edition, 1991.

[11] H. C. Hamaker, "The London-van der Waals attraction between spherical particles," Physica, vol. 4, no. 10, pp. 1058-1072, 1937.
[12] L. Bo, H. P. Zhao, and X. Q. Feng, "Spontaneous instability of soft thin films on curved substrates due to van der Waals interaction," Journal of the Mechanics and Physics of Solids, vol. 59, no. 3, pp. 610-624, 2011.

[13] B. Q. Su, H. S. Hu, C. L. Shen, Y. L. Pan, and G. L. Zhang, Differential Geometry, People's Education Press, 1979.

[14] J. Y. Wu, Biomembrane free energy and the driving forces induced by curvature gradients [Ph.D. thesis], School of Aerospace, Tsinghua University, Beijing, China, 2012. 

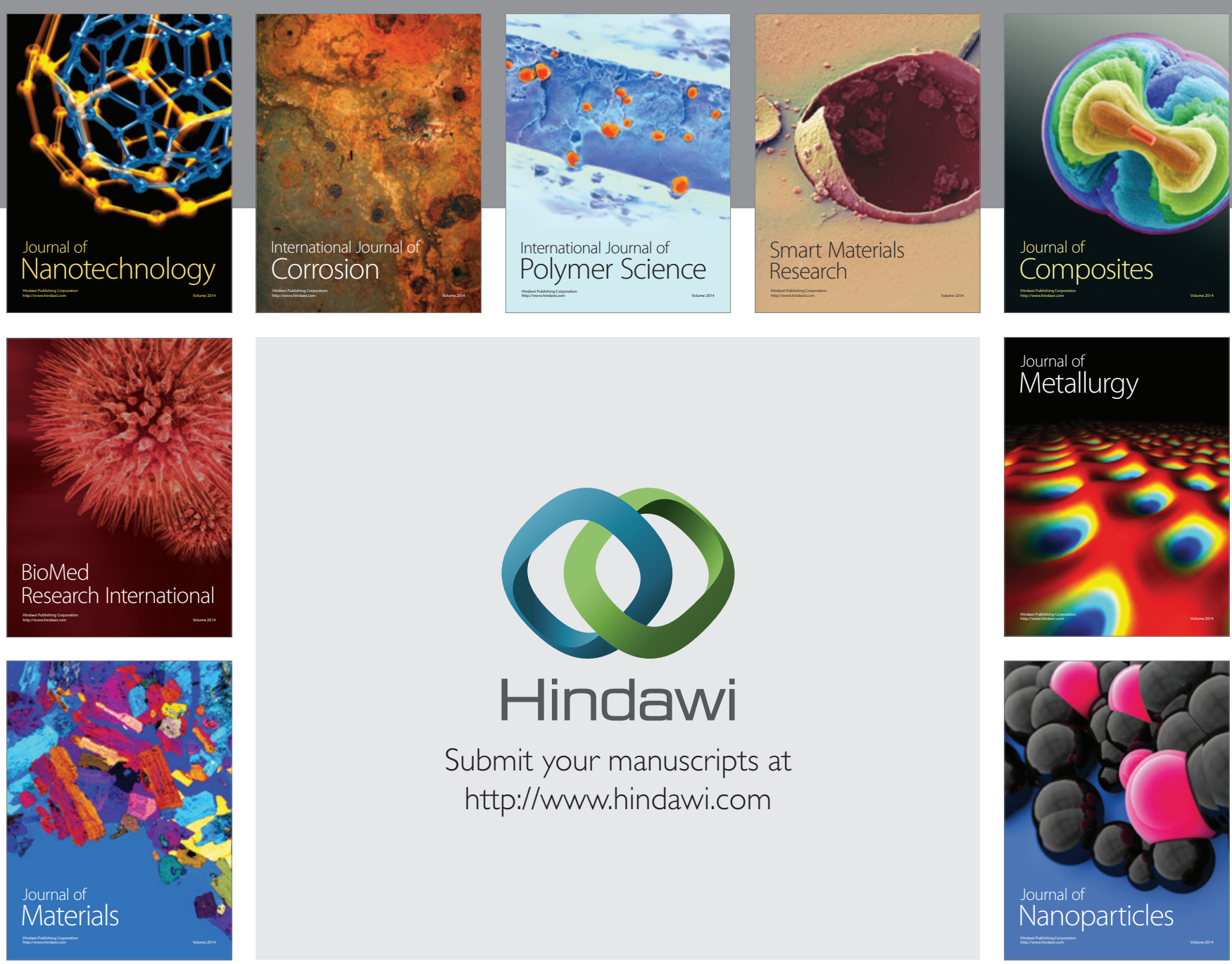

Submit your manuscripts at http://www.hindawi.com
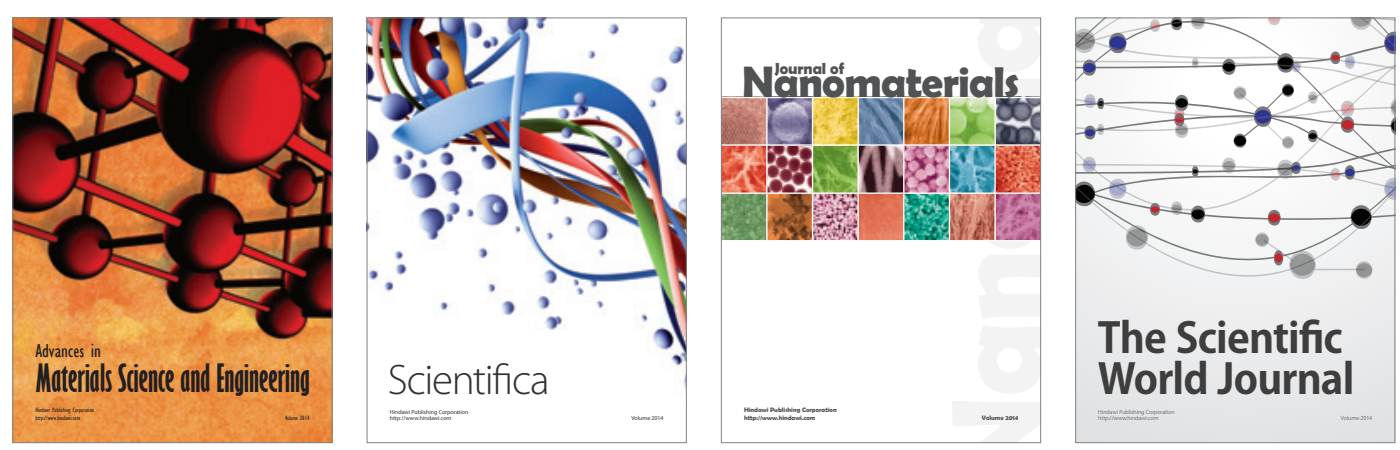

\section{The Scientific World Journal}
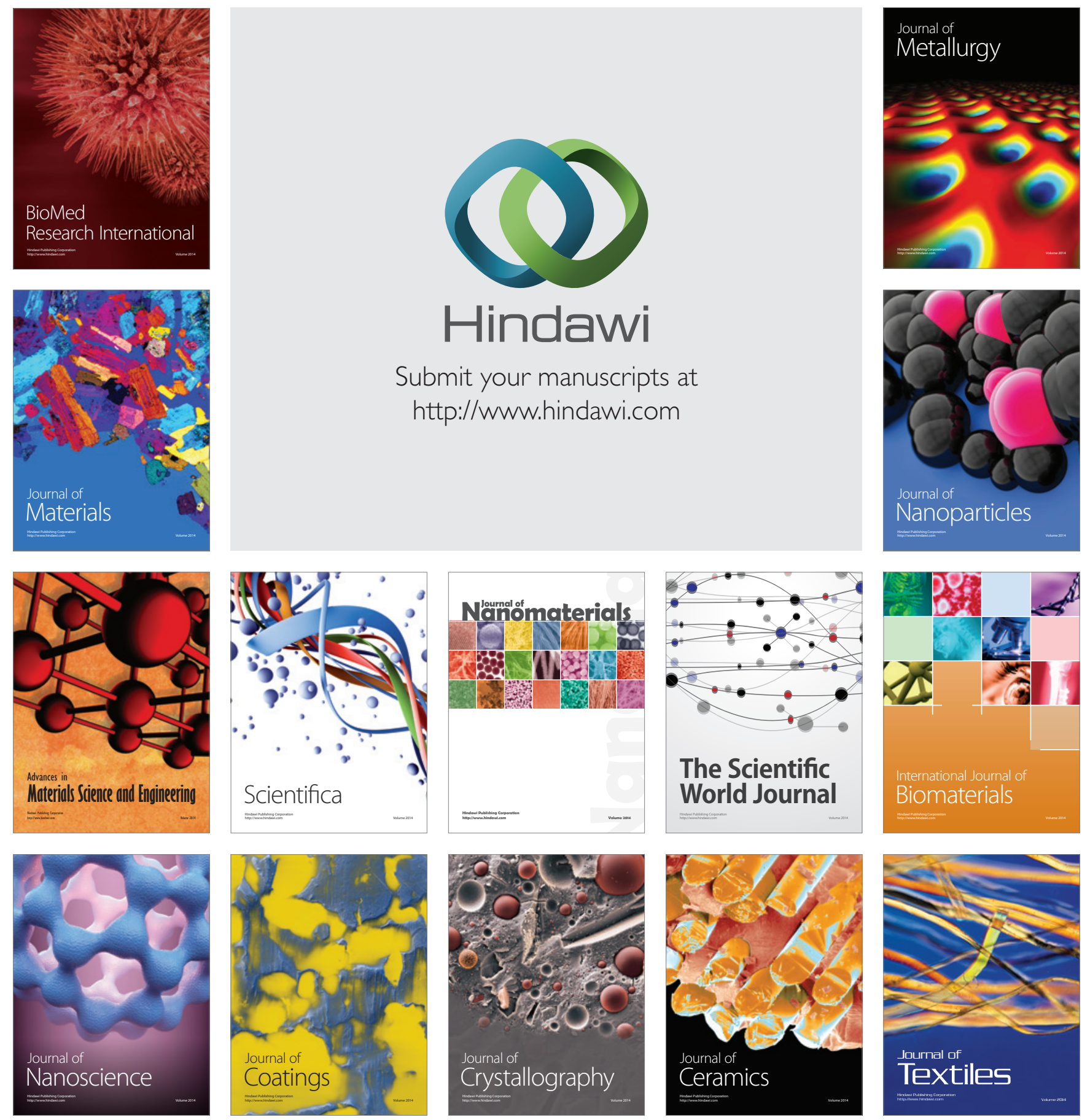